\title{
Two-echelon Emergency Response Problem and Simulation Considering Secondary Disasters
}

\begin{abstract}
It is necessary for subsequent resource distribution planning that get expected relief time considering secondary disasters after natural disaster. The goal of this research is to develop a two-echelon emergency resource distribution model under condition of secondary disasters. Taking minimal maximize relief time as criterion of relief performance, we developed response strategies and simulation model to get the expected value. Numerical studies of this paper presented the result of response strategies.
\end{abstract}

Keywords: secondary disasters, two-echelon vehicle routing model, simulation

\section{Introduction}

China is a country with frequent natural disasters. Some natural disasters, such as earthquakes, floods and other major natural disasters, cause great casualties and property losses. In the process of relief, it is necessity to solve the people's basic living by supplying many substances in the affected areas. After a natural disaster occurred, we should allocate limited supplies in the shortest time. It is of great significance for reducing the loss caused by natural disasters.

After the disaster, how to make the farthest victims obtain relief supplies as soon as possible becomes the one of key index to measure efficiency of relief system. Multi-level material dispatching system is frequently used in practice. That is to say, the relief resource is transported from external areas to disaster relief centers, and to next distribution sites, and finally to victims. However, the secondary disasters, often occur in the rescue process, will affect the material dispatching plan, and ultimately affect the rescue efficiency. For example, road landslide and debris flow after earthquake often hinder the delivery goods to specific implementation

Manuscript received May 2, 2014; accepted September 10, 2014

Han-peng Zhang, Yi Liao, Hui-xia Luo (凶)

Southwestern University of Finance and Economic, Chengdu 611130, China

Email: zhanghanpeng@163.com region. Assumption that all the roads leading to a certain affected area are blocked due to secondary disasters, vehicles equipped with relief supplies are planned at congestion of each road, in order to deliver goods as soon as possible to the victims. Once road is gotten through, vehicles immediately drive to affected areas. When facing above second disasters under the condition of minimizing the delivery time, reasonable goal evaluation has key role for the later materials preparation and adjustment of rescue plan.

In the study, assuming that the relief resource outside transporting to the affected areas need transshipment from local distribution centers, we estimate the performance of relief efficiently based on vehicle routing problem (VRP).

\section{Literature review}

In the existing studies, the research considering one-echelon vehicle routing problem is rich, but research considering multiple-echelon vehicle routing problem is rare. The multi-echelon vehicle routing problem was first proposed by Crainic, Mancini, Perboli and Tadei in 2008. It is still a relatively new research topic at present. There are a few papers studying the question (Crainic, Mancini, Perboli, \& Tadei, 2008a; Crainic, Mancini, Perboli, \& Tadei, 2008b; Hemmelmayr, Cordeau, \& Crainic, 2012; Perboli \& Tadei, 2010; Perboli, Tadei \& Vigo, 2011).

In a typical disaster relief system, the objective function of majority research mostly is the minimum total cost of the rescue. In fact, the last time to receive relief resource is also used to measure the rescue performance. This kind of problem is the minimum maximum vehicle routing problem. As concerning the minimum maximum path problem, Lin and Qi studied the one-echelon vehicle routing problem with $\mathrm{M}$ vehicles which have same capacity, and gave an improved tabu search (Lin \& Qi, 2007; Lin \& Yang, 2012). But this research is also aimed at one-echelon vehicle routing problem.

About emergency study with vehicle routing problem, Liu, He and $\mathrm{Si}$ (2001) studied emergency supplies scheduling problem with more save points, and construct an emergency rescue model under the condition of the earliest emergen- 
cy time and constraints. Wang, Chen, Lu and Wang (2013) proposed a three-echelon emergency supplies collaborative scheduling model under the condition that the relief resource less than the demand in the emergency response, and ultimately gave a greedy algorithm. Zhang, Guo and Li (2007) aimed at road repair after disaster and relief supplies distribution problem, utilized time-space network flow techniques, and constructed a resource allocation and delivery solution which may improve work efficiency in a limit time.

Because of the obvious differences between minimum maximum target and minimum goals, the strategies for performance target in minimum maximum two-stage relief time model are also different. The existing research about the secondary vehicle routing problem considers that there is only one distribution center in the first echelon and several ones in the second echelon. Therefore, this paper tries to develop a new two-echelon VRP model to analyze the scenario of response strategies for secondary disasters after specific natural disaster occur.

\section{Model development}

The mathematical model are as follows:

$$
\begin{aligned}
& \min \lambda^{\overline{\max }} \\
& \text { s.t. } \quad \lambda_{k}^{l} \leqslant \lambda^{\max } \quad \forall k \in V_{s}, \forall l \in V_{n} \\
& \lambda_{k}^{l}=\sum_{i \in V_{c k}} \sum_{j \in V_{c k}} t_{i j}^{k} y_{i j}^{k}+\sum_{j \in V_{c k}} t_{k j}^{k} y_{k j}^{k}+\sum_{j \in V_{c k}} t_{j k}^{k} y_{j k}^{k}+T_{k}^{l} \\
& \forall k \in V_{s}, \forall l \in V_{n} \\
& \sum_{j \in V_{c}} y_{i j}^{k}=\sum_{j \in V_{c}} y_{j i}^{k} \quad \forall k \in V_{s}, \forall i \in V_{s} \\
& \sum_{j \in V_{s}} x_{i j}=1 \quad \forall i \in\left\{V_{s}, V_{n}\right\} \\
& \sum_{j \in V_{c}} y_{i j}^{k}=1 \quad \forall i \in V_{s} \cup V_{c} \\
& T_{k}^{l}=T_{l}+\sum_{k \in V_{s}} t_{l k} x_{l k}+\sum_{k \in V_{s}} \sum_{k^{\prime} \in V_{s}} t_{k k^{\prime}} x_{k k^{\prime}} \\
& \forall k, k^{\prime} \in V_{s}, \forall l \in V_{n} \\
& x_{i j} \in\{0,1\}, y_{i j}^{k} \in\{0,1\}
\end{aligned}
$$

$V_{n}$ is the set of blocked roads; $V_{s}$ is the set of distribution centers in affected areas; $V_{c}$ is the set of victims; $V_{c k}$ is the set of victims of victims which receive relief resource from distribution centers $k ; t_{i j}^{k}$ is the time that the vehicle $k$ in affected area distribution center via victim $i$ to victim $j ; T_{k}^{l}$ is the time from blocked road $l$ to distribution centers $k ; T_{l}$ is the time of road open again; $x_{i j}$ is the integer variable: $x_{i j}=1$, when vehicle travel from the affected area distribution center $i$ (or blocked roads $i$ ) to the affected area distribution center $j$ in the first echelon by vehicle in affected area distribution center $k$; otherwise, $y_{i j}^{k}=0 ; \lambda^{\max }$ is the maximum expectations time that victims receive relief resource; $\lambda_{k}^{l}$ is the time that supplies relief resource from blocked road $l$ to victim through distribution center $k ; t_{l k}$ is the time that transport relief resource form blocked road $l$ to affected area distribution center $k ; x_{l k}$ is the integer variable; $x_{l k}=1$, when vehicle travel form locked road $l$ to the affected area distribution center $k$, otherwise, $x_{l k}=0$; $t_{k k^{\prime}}$ is the time that transport relief resource form $b$ affected area distribution center $k$ to affected area distribution center $k^{\prime} ; x_{k k^{\prime}}$ is the integer variable; $x_{k k^{\prime}}=1$, when vehicle travel form affected area distribution center $k$ to the affected area distribution center $k^{\prime}$, otherwise, $x_{k k^{\prime}}=0$.

Function (1) denotes the maximum time of minimizing obtaining relief resource for victims; function (2) says time that all the victims receive relief resource is less than or equal to the last time of obtaining relief resource for victims; functions (3) and (7) denote that time of receiving relief resource for victims include time of road repair, the delivery time of first echelon, and the delivery time of second echelon; function (4) denotes that vehicle starting from distribution center of the second echelon will return the same center; function (6) denotes that the demand of victims is supplied only by one distribution centers in the affected areas.

If $T_{k}^{l}=0$, this model is the typical minimum maximum two-chelon vehicle routing problem. But as a random variable, the opening time of blocked roads $l$ will affect the whole rescue performance. If there are $\mathrm{n}$ roads, one can assume that opening time of each road conforms to the uniform distribution of $[0, m]$. Only consider the integer value, the combination of the $n$ road opening time is $m^{n}$. If victims conform to the uniform distribution, each local distribution site also conforms to the uniform distribution, and the distance between blocked roads and affected area centers is equal, this problem will be simplified. But the routes are different with different open road time combination scenario, which increase complexity. So, as for multiple road system, simulation technology is an effective means for forecasting relief performance.

\section{Response strategy and simulation steps}

Assuming that each vehicle of blocked roads only distribute integer times of the demand of the regional distribution center, which means each blocked region supply the whole relief resource for certain distribution center, or not. In the same time, assuming that relief resource delivered by each affected regional distribution center equal to the number of victims.

Repair time submit to the uniform distribution of $[0, m]$. Once opened, the reasonable response strategies are as follows.

(1) Once the blocked road is repaired, it is a reasonable 
path by which relief resource is dispatched to the nearest distribution center.

(2) In the process of dispatching next node, if other road is also opened again, the reasonable path of the two vehicles in first echelon can be made by minimizing system relief time. If there is more than one, the rest can be done in the same manner.

(3) After relief resource is delivered to the affected area distribution center, if it is still only one congested road opened again, the path to victims is made by minimizing delivery time of this center.

(4) Suppose that there are $n$ roads leading to the disaster area opened again and vehicles in first echelon all dispatch relief resource to the several affected area distribution centers respectively, the rule is that the minimum time of arrival at next distribution center is priority for all vehicles in first echelon.

Simulation steps:

(1) According to the uniform distribution, multiple roads opening time is randomly formed;

(2) According to the corresponding road opening time $T_{k}^{l}$, the set is determined of the affected area distribution centers that every vehicle in first echelon should dispatch;

(3) According to the two levels multi-depot vehicle routing problem algorithm, optimize the distribution path from affected area distribution center to victims;

(4) Obtain every victim's time of gaining relief resource, and look for the maximum;

(5) Iterate and stop when to run a certain number of times.

\section{Numerical experimentation}

This article selects A-n32-k5 in capacitated vehicle routing problem (CVRP) points of library of VRP case library as an example. There are 32 nodes as the victims, each point's demand is 1 . The coordinates of increasing 4 affected regional distribution centers are $(10,40),(50,30),(40,60),(70)$ respectively. So each distribution center should deliver relief resource to 8 victims. Assume that there are two roads to affected area and the coordinates is $(0,50)$ and $(100,50)$. Opening time conforms to the uniform distribution $[0,45]$, according to the response strategy and simulation steps proposed in this paper, the ultimate time-demand distribution chart of gaining relief resource can be obtained, and is as followed in Figure 1.

From Figure 1, as the opening time delay, the time of obtaining relief resource for the victims also will be delayed. But there is no proportional relationship between the two variables. Moreover, the different opening time of the two roads also does not produce symmetry results. The main reason is that according to the different opening time scenario, affected area distribution centers will optimize their own distribution path in the process of rescue, and modify the last result.

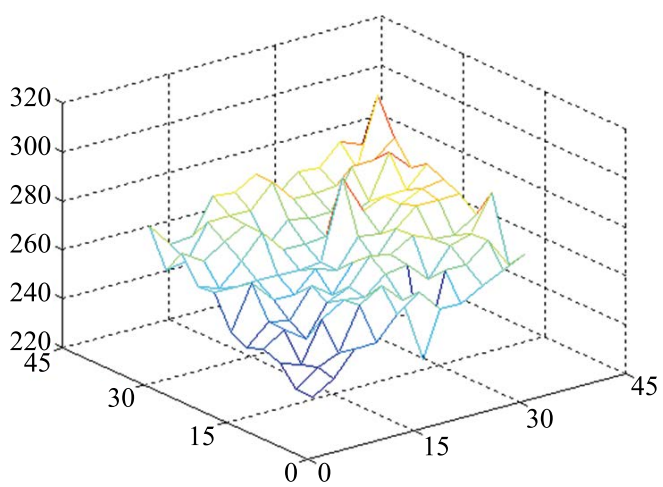

Figure 1. The simulation results.

\section{Conclusion}

This paper proposes the minimum maximum two-echelon relief distribution model, formulates the response strategy and simulation steps, and finally uses numerical experiments to simulate and analyze the proposed model and strategy. This model provides decision making ideas and auxiliary support for relief resource plan and scheduling changes after secondary disaster.

Acknowledgement This research work was supported by the National Natural Science Foundation of China (Nos.70801049; 71201128; 71302187) and the Ministry of Education of Humanities and Social Science project (No.14XJC630010) in China.

\section{References}

Crainic, T., Mancini, S., Perboli, G., \& Tadei, R. (2008a). Multi-start heuristics for the two-echelon vehicle routing problem. In P. Merz \& J.K. Hao (Eds). Proc. Evolutionary computation in combinatorial optimization: 11th European conference, 179-190

Crainic, T., Mancini, S., Perboli, G., \& Tadei, R. (2008b). Clustering-based heuristics for the two-echelon vehicle routing problem, CIRRELT, Montréal, CIRRELT-2008-46. Retrieved from http:// www.cirrelt.ca/DocumentsTravail/CIRRELT-2008-46

Hemmelmayr, V., Cordeau, J. F. O., \& Crainic, T. (2012). An adaptive large neighborhood search heuristic for two-echelon vehicle routing problems arising in city logistics. Computers \& Operations Research, 39, 3215-3228

Lin, X., \& Qi, H. (2007). Min-max vehicle routing problemtabu searchheuristics. Systems Engineering, 25(1), 49-52

Lin, X., \& Yang, C. (2012). Min-max vehicle routing problem based on ant colony algorithm. Journal of PLA University of Science and Technology (Natural Science Edition), 13(3), 336-341

Liu, C., He, J., \& Si, J. (2001). The study on optimimal model for a kind of emergency materials dispatch problem. Chinese Journal of Management Science, 9(3), 29-36 
Perboli, G., \& Tadei, R. (2010). New families of valid inequalities for the two-echelon vehicle routing problem. Electronic Notes in Discrete Mathematics, 36, 639-646

Perboli, G., Tadei, R., \& Vigo, D. (2011). The two-echelon capacitated vehicle routing problem: models and math-based heuristics. Transportation Science, 45(3), 364-380
Wang, J., Chen, J., Lu, Y., \& Wang, M. (2013). Study on collaborative scheduling method under emergency at sea based on demand chain. Application Research of Computers, 30(6), 1727-1730

Zhang, Y., Guo, X., \& Li, J. (2007). Combinatorial optimization algorithm of rapid road repair and material distribution after disaster. Journal of Traffic and Transportation Engineering, 7(2), 117-122 